\title{
EL PRACTICUM DEL GRADO EN INFORMACIÓN Y DOCUMENTACIÓN: LA EXPERIENCIA EN LA UC3M
}

\author{
Marina Vianello Osti* \\ Departamento de Biblioteconomía y Documentación. Universidad Carlos III de Madrid. \\ Belén Pérez Lorenzo** \\ Departamento de Biblioteconomía y Documentación. Universidad Carlos III de Madrid. \\ Ana R. Pacios Lozano *** \\ Departamento de Biblioteconomía y Documentación. Universidad Carlos III de Madrid.
}

\begin{abstract}
Resumen: Se expone el proceso seguido en la implantación del Practicum en el grado en Información y Documentación de la Universidad Carlos III de Madrid y se presenta una herramienta para su seguimiento y evaluación. A partir de una revisión de la literatura sobre el concepto de competencia y su importancia en la educación superior, y de las características que deben presentar las prácticas curriculares para una correcta interrelación con el plan de estudios, se propone una matriz de evaluación para orientar su realización y evaluación y unas encuestas para conocer la percepción y el resultado de la experiencia. Analizadas las encuestas y evaluados informes y memorias de dos cursos académicos podemos considerar que la experiencia ha sido positiva y que la percepción de tutores y alumnos ha sido buena. Sin embargo es posible mejorar algunos aspectos intensificando la relación y el intercambio de información entre los diferentes actores que intervienen en el Practicum.
\end{abstract}

Palabras clave: Practicum; grado en Información y Documentación; competencias; matriz de evaluación; encuestas de satisfacción.

Title: THE PRACTICUM IN THE DOCUMENTATION AND INFORMATION SCIENCE BACHELOR'S DEGREE PROGRAMME: THE EXPERIENCE AT UNIVERSIDAD CARLOS III, MADRID.

Abstract: This study shows the process followed for the introduction of the Practicum in the Documentation and Information Science Bachelor's Degree program at Universidad Carlos III de Madrid and presents a tool for its monitoring and evaluation. From a review of the literature on the concept of competence and its importance in higher education, as well as the necessary features a Practicum must have for it to be properly interrelated with program curriculum, an evaluation matrix is proposed to guide its use and evaluation, together with surveys to determine perception and results of the experience. By analyzing the results of the survey and evaluating reports from the past two years that the Practicum has been in place, we determine that the experience has been positive and that both tutors and students alike perceive it in a favorable light. Nevertheless, it is possible to improve in some aspects by strengthening the relationship and exchange of information between the different actors who intervene in the Practicum.

Keywords: Practicum; Bachelor's Degree in Documentation and Information Science; competences; evaluation matrix; satisfaction surveys.

\section{INTRODUCCIÓN.}

Las prácticas académicas externas en unidades de información (bibliotecas, archivos y centros de documentación, en su mayoría, además de otras como empresas de gestión documental o gestión de contenidos) suponen una primera aproximación a las tareas del profesional. A la vez permiten al alumno aplicar y complementar los conocimientos adquiridos en su formación académica. A ello hay que añadir que los alumnos pueden ser conscientes de los continuos cambios en el entorno profesional, lo que contribuye a evitar el desfase entre su formación y las futuras responsabilidades profesionales, aspecto detectado en algunos trabajos como el de Westbrock y Fabian (2010).

De ahí que las prácticas académicas hayan adquirido gran relevancia y necesidad en el actual contexto universitario, globalizado y altamente competitivo, y así lo refleja la profunda reforma llevada a cabo en España con la nueva estructuración de las enseñanzas y títulos universitarios oficiales, concretada en el Real Decreto 1393/2007, para adaptarlos al Espacio Europeo de Educación Superior (en adelante EEES), que supuso la puesta al día del Practicum también en los nuevos planes del título de Grado en Información y Documentación.

\footnotetext{
* mvianell@bib.uc3m.es

**mperez@bib.uc3m.es

***arpacios@bib.uc3m.es
}

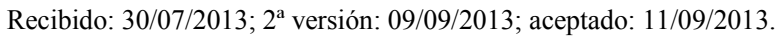

VIANELlO OSTI, M.; PÉREZ LORENZO, B. y PACIOS LOZANO, A.R. El Practicum del grado en Información y Documentación: la experiencia en la UC3M. Anales de Documentación, 2013, vol. 16, nº 2.

Disponible en: http://dx.doi.org/10.6018/analesdoc.16.2.180381 
Las precedentes titulaciones (diplomaturas y licenciaturas) han venido incluyendo prácticas externas curriculares de carácter obligatorio. Esta característica, más bien poco habitual, permite afirmar que, en el caso del grado en Información y Documentación, se cuenta con una consolidada "cultura del Practicum" (Zabalza Beraza, 2011). Y, por tanto, con una experiencia que ha permitido repensar el mismo. Prueba de ello es que en el Libro Blanco del Título de Grado en Información y Documentación (ANECA, 2004) se propuso, dentro de los contenidos comunes obligatorios (de carácter troncal), la asignatura de Practicum con una carga de 12 créditos, lo que supone un total de 300 horas de trabajo del alumno.

La puesta en marcha del Practicum requiere de procedimientos que garanticen su calidad. Es en esta línea en la que se enmarca este trabajo cuyo objetivo es, además de describir el proceso seguido para el nuevo planteamiento de la asignatura en su adaptación al EEES, presentar una herramienta para su seguimiento y evaluación. Los puntos de partida para su diseño han sido el concepto de competencia y la experiencia acumulada en los estudios anteriores. Se presentan los resultados y las opiniones que han merecido los primeros dos cursos de aplicación del modelo, recabadas a través de las evaluaciones y las encuestas realizadas.

\section{REVISIÓN DE LA LITERATURA.}

La literatura relativa a la evolución de las prácticas de estudiantes en bibliotecas ha sido objeto de una buena revisión reciente por parte de Sargent; Becher y Klingberg (2011).

Las prácticas de los estudiantes, tanto las curriculares como las extracurriculares, han venido siendo tema de investigación y debate a lo largo de los años. Hay numerosos estudios al respecto en los que se dan a conocer las características de las mismas en determinadas universidades y titulaciones, a la vez que se presentan propuestas de cómo se deben organizar y evaluar. Recordamos los trabajos de Moneda y Peis (1995), Carrizo Sainero (1997), Chain Navarro, Múñoz Cañabate y Blanco Faura (2009), así como el más reciente de Espelt y Maña (2011). En todos se pone de manifiesto la preocupación por una adecuada interrelación de las prácticas con el plan de estudios del que forman parte. Otros, como Fernández (1998), han insistido en dar al Practicum un papel hegemónico señalando su valor y las ventajas que conlleva para la universidad: apertura de nuevas líneas de investigación, reorientación de los planes de estudios y canalización del diálogo entre la sociedad y la universidad.

Sobre su evaluación, la recomendación generalizada, y así es como se viene haciendo en muchas universidades, es que se realice desde diferentes perspectivas. Algunas guías ofrecen propuestas de interés en este sentido (Baird, 2008). Para que la evaluación sea lo más objetiva posible se debe involucrar a los estudiantes, profesores-tutores y a los profesionales que les acogen en las empresas y organizaciones y actúan como supervisores (Beard, 2007). Se trata de que exista una interacción y feedback constante entre todos los actores implicados para que, con los datos que proporciona, se pueda llevar a cabo un análisis, discusión y desarrollo de acciones en consonancia.

En España, con la introducción del Practicum en todos los grados, se han realizado estudios para la reflexión, acción y toma de decisiones en este contexto. Estos aspectos se pueden ver en monográficos dedicados al tema, ejemplo de la Revista de Educación (2011), así como en congresos y seminarios en los que ha sido uno de los temas centrales a debatir. Prueba de ello es el "Symposium Internacional sobre el Practicum" (2011), celebrado en la Universidad de Vigo, cuyo tema central fue el compromiso con la calidad de las prácticas y en el que se intercambiaron experiencias y propuestas de numerosas titulaciones. En particular, para el título de Grado en Información y Documentación también se ha hecho hincapié en la necesidad de actualizarlo y adecuarlo a las demandas del EEES (Osuna, 2008).

En la conferencia de la IFLA de 2011 se presentaron diferentes trabajos relativos a la formación práctica de los futuros profesionales de la información y la documentación en los que se muestra el panorama en distintos países como Perú (Jara de Súmar y Talavera Ibarra, 2011), o incluso en un ámbito territorial más amplio como el latinoamericano (Araki y de la Vega, 2011). En todos ellos se dan recomendaciones para que las prácticas ayuden a formar profesionales competentes y se abran paso en el mercado laboral.

Aunque el Practicum en España ha sido analizado desde diferentes perspectivas, faltan, por ejemplo, valoraciones realizadas por los estudiantes. En las escasas experiencias que se han dado a conocer, los estudiantes le han otorgado una alta valoración (Mendo et al., 2001) dado que no hay ningún aspecto que fuera puntuado por debajo de 5 como media. Entre los aspectos más valorados destacan la importancia concedida al Practicum dentro del plan de estudios y el interés de los alumnos por los centros ofertados. En relación con las tareas desempeñadas, aunque el 79,9\% 
manifestaron que eran específicas de unidades de información, los que opinaron lo contrario argumentaron que eran tareas no relacionadas con la profesión, tenían poco que ver con los conocimientos adquiridos o eran de tipo muy repetitivo. El último trabajo en esta línea es el realizado por Pacios (2013), aunque centrado en un aspecto concreto, el proceso de inducción.

Los trabajos relacionados con experiencias de evaluación de las prácticas en España son más bien escasos. Existe unanimidad en el uso de una serie de herramientas utilizadas con esta finalidad (memorias, informes de los responsables de los centros, etc.). Hasta la fecha, y en el caso particular del grado en Información y Documentación, los trabajos se han centrado más en la definición de las competencias que se deben trabajar.

Por otra parte, también hay que indicar que a lo largo de todos estos años de experiencia, las universidades cuentan con datos procedentes de su evaluación que han venido utilizando para planificar y mejorar cada nuevo curso de prácticas. Esta información, de carácter interno y manejada por los responsables de las prácticas, es de gran importancia para tomar decisiones relacionadas con su mejora continua.

\section{EL CONCEPTO DE COMPETENCIA Y SU IMPORTANCIA EN LA FORMACIÓN SUPERIOR.}

De la revisión de la literatura se desprende la importancia que reviste la aplicación del concepto de competencia al diseño curricular de los nuevos grados, donde se trata de proporcionar al estudiante una formación que, sacrificando algo del academicismo tradicional, oriente los conocimientos hacia resultados que estrechen la relación entre universidad y empresa, entre oferta y demanda. (Tejada Artigas, 2006; Fernández-Salinero, 2006; Mano, 2009).

El término competencia surge inicialmente en un contexto vinculado a la formación y al empleo, no es sencillo de describir debido a su carácter polisémico y que, desde su aparición, ha ido ampliándose dando lugar a una gran proliferación de definiciones no siempre convergentes (Olaz Capitán; Ortiz García y Sánchez-Mora Molina, 2011). Su origen está en el ámbito empresarial donde se puso el acento en la insuficiencia de la cultura académica y los títulos de estudios para predecir el éxito profesional, señalando así el camino para la identificación de otros factores condicionantes de un buen desempeño profesional (Mcclelland, 1973).

A partir de esta constatación los esfuerzos de los investigadores se han orientado a identificar las competencias que posibilitan el éxito profesional. Consideradas características intrínsecas al individuo relacionadas con un rendimiento eficiente se distingue entre unas competencias específicas, que se relacionan directamente con la utilización de conceptos, teorías o habilidades propias de una profesión, y las competencias transversales, también llamadas genéricas, que son comunes a la mayoría de las profesiones y que se relacionan con la puesta en práctica integrada de aptitudes, rasgos de personalidad, conocimientos y valores adquiridos (Boyatzis, 1982, p. 23).

La sucesión de propuestas de clasificación de las competencias (Hay/McBer, 1961) acaban poniendo de manifiesto que las competencias solo se manifiestan en un contexto específico (Spencer y Spencer, 1993). En definitiva, como escribe Tejada Fernández (2005): "Las competencias sólo son definibles en la acción”, no pertenecen ni al ámbito del saber ni del saber-hacer, sino que estriban en la movilización de recursos, en su utilización. Las competencias combinan entre sí conocimientos, habilidades, actitudes, motivaciones y destrezas, pero su manifestación solo se produce en el contexto, cuando una persona realiza una tarea concreta en el marco de un trabajo específico, así que, tan esencial es su definición como su adquisición, aplicación y verificación.

La voluntad de alcanzar una integración entre la formación superior y el mercado de trabajo ha trasladado estos conceptos a la universidad donde se plasma en la formulación del EEES. Este enfoque, adoptado de inmediato por la universidad española, a pesar de dar lugar a un debate entre los especialistas favorables a su adopción en los estudios universitarios (Pimienta Prieto, 2011; Ribes Iñesta, 2011; Salvador Oliván, 2011) y otros discrepantes (Carabaña Morales, 2011; Castro Morera, 2011), conforma las directrices para el diseño de los nuevos grados. Desde esta perspectiva los nuevos planes de estudio implican por un lado a la universidad que tiene que proporcionar los conocimientos y desarrollar las capacidades de los alumnos y, por el otro, al mundo del trabajo que tendrá que emplearlos. La cuestión ahora estriba, además de saber definir cuáles son las competencias adecuadas a cada perfil profesional, en identificar qué tipo de formación es la idónea para lograrlas.

En este sentido el Proyecto Tuning (González y Wagenaar, 2006) - impulsado por las universidades europeas para propiciar la convergencia al EEES - pone de manifiesto que el diseño de los títulos universitarios se debe realizar en función de las actividades que los egresados estarían en condición de desempeñar, y la evaluación del aprendizaje no debe basarse únicamente en los conocimientos sobre la materia, sino que debe sustentarse en la consecución de unas 
competencias que representen una combinación dinámica de elementos personales, desarrollados a partir de un programa educativo que asegure la inserción laboral exitosa de los titulados.

Por su parte el Proyecto DeSeCo (Definition and Selection of Competencies) ${ }^{\mathrm{i}}$ encargado por la Oficina de Educación de la OCDE indica que la construcción de las competencias debe hacerse a través de la combinación de habilidades cognitivas y prácticas, conocimiento (incluyendo el conocimiento tácito), motivación, valores, actitudes, emociones y otros componentes sociales y conductuales. Tanto la capacidad como la acción efectiva implican la movilización de conocimiento, habilidades cognitivas y prácticas, así como componentes sociales y de comportamiento no cognitivo, tales como actitudes, emociones, valores y motivaciones (Rychen y Salganik, 2002).

Así pues, resulta patente y el Proyecto Tuning lo señala, que las universidades no deben transferir solo conocimientos consolidados, sino también una variedad de competencias genéricas. Para facilitar este proceso se aconseja distinguir entre competencias específicas y genéricas, organizándose estas últimas en:

a) instrumentales (habilidades cognoscitivas, capacidades metodológicas, destrezas tecnológicas y destrezas lingüísticas), b) interpersonales (capacidades individuales -expresión de sentimientos, habilidades de crítica y de autocrítica; destrezas sociales- capacidad de trabajar en equipo, expresión de compromiso social o ético), y c) sistémicas (destrezas y habilidades relativas a los sistemas como totalidad).

Estas indicaciones finalmente se reflejan en los libros blancos llevados a cabo por las universidades españolas, apoyadas por la ANECA, que hacen de las competencias el elemento imprescindible para el diseño de los títulos de grado adaptados al EEES. Y el Practicum, desde esta perspectiva, constituye sin duda el entorno privilegiado para la transferencia de competencias, ya que representa la convergencia entre el mundo formativo y el laboral, poniendo en contexto todas las habilidades y capacidades adquiridas con las diferentes asignaturas.

\section{EL PRACTICUM DEL GRADO EN INFORMACIÓN Y DOCUMENTACIÓN EN LA UNIVERSIDAD CARLOS III.}

La puesta en marcha en la Universidad Carlos III del nuevo grado, en el año académico 2008/2009 nos ha dado la oportunidad de reflexionar acerca de su fundamentación teórica, con el objetivo de dotarlo de una estructura que haga de esta experiencia un momento clave para la formación del estudiante, propiciando su reflexión acerca de la aplicación en un contexto real de los conocimientos adquiridos en la carrera. Siguiendo los modelos de aprendizaje basados en la experiencia (Zabalza, 2011) hemos profundizado, primero, en el análisis de su organización, para luego detenernos en las competencias que moviliza, los actores que implica y su ámbito de verificación para definir una matriz de evaluación y, a partir de allí, poder diseñar los instrumentos apropiados para orientar su desarrollo.

\subsection{La organización del Practicum.}

El punto de partida para diseñar la organización del Practicum ha sido el análisis de la experiencia anterior. Después se han definido los elementos de innovación necesarios para construir una nueva propuesta que optimice la formación práctica integrándola con la formación académica.

El Departamento de Biblioteconomía y Documentación de la Universidad Carlos III de Madrid, al que está adscrito el Grado en Información y Documentación, cuenta con una larga trayectoria de gestión de prácticas académicas, concretamente desde el año 1989 en que se implanta en la UC3M la Diplomatura en Biblioteconomía y Documentación.

Los objetivos fijados para las "Prácticas en Unidades de Información" de la antigua diplomatura y licenciatura de nuestra Universidad eran claros: "aplicación en unidades de información de los conocimientos teóricos y prácticos adquiridos en los correspondientes cursos (...)" (UC3M-Fichas Asignatura). Desde ese año, cerca de 500 entidades han venido colaborando con las titulaciones de Información y Documentación de la Universidad acogiendo a nuestro alumnado en estancias de prácticas. En el año 2008/2009, con el fin de evaluar, mejorar y acercar las prácticas a la nueva filosofía de los grados se inicia un proyecto de innovación docente que nos permite, a través de encuestas a estudiantes y a tutores de centros, profundizar en las expectativas, logros y problemas que suscita esta asignatura. Finalmente el Practicum en el grado en Información y Documentación inicia su andadura en el curso 2011/2012.

De acuerdo con el Reglamento de prácticas externas de la Universidad Carlos III de Madrid, aprobado en 2010, su tutorización, al igual que sucedía en la antigua diplomatura, está a cargo del Departamento de Biblioteconomía y Documentación y su coordinación depende de la Facultad de Humanidades, Comunicación y Documentación. En el nuevo grado, según consta en la Memoria verificada por la ANECA el 28 de mayo de 2008, el Practicum es 
obligatorio y su valor en ECTS es de 18. Suponen la suma de los créditos que se dedicaban a las prácticas en la diplomatura y la licenciatura y supera la carga sugerida en el Libro Blanco que propone un Practicum de 12 ETCS, dejando patente la importancia que se atribuye a esta experiencia formativa en nuestra Universidad. La consecución de estos créditos que representan 450 horas de trabajo del alumno se distribuye entre un plan de formación de 375 horas de prácticas, a razón de cinco horas diarias, de lunes a viernes en un centro colaborador a lo largo de tres meses, y las horas que el alumno tiene que dedicar en su casa a verificar sus conocimientos para poderlos aplicar en el trabajo y las reflexiones sobre la experiencia que finalmente se tendrán que plasmar en la memoria final.

La realización de las prácticas en una única estancia en el segundo cuatrimestre del cuarto curso constituye un cambio importante con respecto a las prácticas anteriores. En la diplomatura las estancias duraban un mes y tenían lugar en $\operatorname{los}$ cursos $2^{\circ}$ y $3^{\circ}$, y en la licenciatura en $2^{\circ}$. Ahora se supera esta fragmentación satisfaciendo una de las quejas afloradas en el proyecto de innovación docente antes mencionado, donde las encuestas revelaban que la gran mayoría de los estudiantes y de los tutores profesionales opinaba que la insuficiente duración de las practicas frustraba el desarrollo de algunas competencias e impedía una buena integración en la actividad de la organización, ya que cuando el alumno había conseguido socializar y asimilar los procedimientos se le acababa la estancia.

Este cambio ha hecho necesario revisar los perfiles de los centros que colaboraban con nosotros: bibliotecas públicas, escolares, especializadas, universitarias, centros de documentación de todo tipo, archivos... Antes, la Comisión Organizadora del Practicum los clasificaba en función del grado de complejidad de las tareas requeridas y asociaba al curso más apropiado. El objetivo era asegurar que los alumnos desempeñaran actividades apropiadas a la formación alcanzada y conseguir un buen aprovechamiento de la estancia.

Sin embargo, ahora los centros donde solo se pueden realizar tareas básicas se han excluido. La Comisión Organizadora del Practicum ha tenido que seleccionar solo aquellos que permiten a los alumnos una visión amplia de la profesión y la realización de una importante variedad de tareas, amén de una calidad de seguimiento apropiada que les lleve a poner en juego el mayor número posible de conocimientos y competencias rotando por sus servicios, favoreciendo su implicación en algún tipo de proyecto. Esta selección ha sido posible revisando las encuestas y las memorias de los antiguos alumnos y manteniendo conversaciones y contactos con los responsables de los servicios y los tutores profesionales, asegurando así su implicación.

Pero el cambio no conlleva solo aspectos positivos. Antes, la realización de las primeras prácticas en segundo de la diplomatura constituía un elemento motivador para los alumnos que con esta incursión temprana en el mundo laboral podían imaginar su futuro y reafirmarse en el acierto de los estudios elegidos. Ahora, perdido este estimulo, intentamos compensarlo organizando -como parte integrante de algunas asignaturas o como actividades complementarias- visitas a centros que permitan conocer a los estudiantes diferentes realidades profesionales. Además, hay que decir que el modelo antiguo era mucho más fácil de asumir por los alumnos que trabajan. En la actualidad este colectivo encuentra muchas dificultades para compaginar las prácticas con sus actividades y esto nos obliga a buscar para cada uno de ellos soluciones personalizadas que en un principio no se habían previsto.

Por lo que se refiere a la localización de los centros colaboradores y su selección, responsabilidad de la Comisión de Prácticas formada por el vicedecano o director del grado y los profesores designados como tutores académicos, se sigue manteniendo en el nuevo grado la estructura organizativa anterior, así como los procedimientos de intercambio de información entre la Comisión y el centro. La novedad está en la labor del tutor académico que ahora se refuerza con unas tutorías grupales de carácter informativo y con la plataforma virtual para proporcionar a los alumnos materiales y facilitar los intercambios de opiniones (chat, foros).

Para la asignación de los alumnos a los centros se realizaba un sorteo, previa elección por parte de los mismos después de haber conocido el perfil de las organizaciones y de las tareas que desarrollarían en ellas. Actualmente, siguiendo la normativa de la Universidad, se ha sustituido el sorteo por un criterio basado en los resultados académicos, que prioriza la elección de los alumnos que han obtenido mejores notas.

\subsection{Las competencias del Practicum y su verificación.}

En los anteriores estudios la evaluación de las prácticas se basaba en la redacción de una memoria por parte del alumno y en un informe del tutor del centro. Las competencias no estaban definidas y su adquisición era evaluada siguiendo unos criterios intuitivos.

En el Libro Blanco del Grado en Información y Documentación, a pesar de que se ratifica su importancia, quizás por ser una materia ya obligatoria en los estudios anteriores, no se encuentra una formulación explícita de las 
competencias asociadas y solamente se señala que contribuirá a desarrollar en mayor o menor medida todas las competencias específicas y genéricas ya desarrolladas en las otras materias (ANECA, 2004, p. 99).

En cuanto a la evaluación del Practicum se fijan tres aspectos para su verificación: el control de la asistencia $(10 \%)$, la evaluación del tutor responsable (50\%) y la memoria del alumno (40\%). Estos aspectos se mantienen en la Memoria del Grado verificada por la ANECA, en cambio, se fijan cinco competencias que el alumno debe alcanzar en la asignatura a través de la "Iniciación al desempeño de la profesión de especialista en información y documentación en el ámbito de instituciones públicas o privadas dedicadas, íntegra o parcialmente, a la selección, tratamiento y difusión de información sin importar los contenidos o formatos que esta adopte”. Las competencias son:

- Conseguir una visión global y directa del funcionamiento de un centro de información

- Adquirir la capacidad necesaria para el desempeño de una labor profesional en el marco de los servicios propios de bibliotecas, archivos o centros de documentación

- Conocer y familiarizarse con las rutinas de trabajo en los procesos de recuperación, localización, tratamiento y difusión de información

- Saber trabajar en equipo, integrándose en un grupo o colectivo profesional del área

- Atender directamente a los usuarios en sus necesidades de información

Se trata de unas competencias de carácter general que necesitan ser contextualizadas y cuantificadas para transformarse en una guía para la realización y evaluación del Practicum. Para eso el primer paso ha sido disgregar estas competencias generales en competencias específicas y competencias transversales. Las competencias específicas surgen del análisis de las habilidades asociadas a las asignaturas del plan de estudios y son las que el alumno debe haber adquirido a lo largo de la carrera. Las competencias transversales, que deberá afianzar el alumno, se han articulado en instrumentales (herramientas para el aprendizaje y la formación), interpersonales (capacidades que permiten mantener una buena relación social) y sistémicas (relacionadas con la gestión de la totalidad de la actuación), están relacionadas con el desarrollo personal, y no dependen de un ámbito temático o disciplinario, sino que penetran en todos los dominios de la actuación profesional y académica (ver Tabla I).

Cada competencia específica y transversal se asocia a sus indicadores, que son las actividades o situaciones que permiten su evaluación, y se identifican los actores implicados en el proceso, su participación, sus necesidades de información y sus responsabilidades (ver Tabla II).

Este punto es muy importante ya que en el Practicum el tradicional binomio docente/estudiante se amplía con la participación de otras figuras que lo hacen posible: el estudiante, el equipo organizador de las prácticas, el tutor académico y el tutor del centro. El estudiante necesita conocer qué se espera de él, cuáles son las competencias que tendrá que demostrar poseer y a través de qué actividades y actitudes tendrá que hacerlo, tanto en el centro donde lleve a cabo la experiencia, como en la elaboración de la memoria que tendrá que entregar una vez finalizada las prácticas.

La Comisión del Practicum, responsable de tramitar los convenios de colaboración, debe conocer todas las actividades relacionadas con las competencias a desempeñar en la práctica para asegurarse de que los centros seleccionados sean idóneos y permitan al alumno desempeñar las competencias fijadas a través de un conjunto suficiente de actividades y situaciones.

El tutor académico tiene que conocer las características de los centros y elaborar, en colaboración con el tutor del centro, el programa de trabajo de los estudiantes con arreglo a las competencias definidas. Es su responsabilidad orientar a los alumnos en el proceso de asignación de los centros. Les informa del funcionamiento de las prácticas, de sus obligaciones y de los criterios de evaluación. Asesora y apoya tanto al alumno como al tutor profesional y realiza un seguimiento permanente. Es responsable de evaluar la memoria de los alumnos y realizará la evaluación final, teniendo en cuenta también la evaluación realizada por el tutor profesional. En cuanto a la memoria, lo que se persigue con su realización es la reflexión. Lejos de querer realizar una evaluación basada en la exhaustividad de la información aportada, nos hemos esforzado para diseñar un instrumento que obligue a un ejercicio de análisis y le permita sacar unas conclusiones acerca de la experiencia realizada que puedan ser punto de partida para futuras experiencias.

El tutor profesional es responsable de la inducción y de informar al alumno de cuáles serán sus tareas y responsabilidades con el centro. Dirigirá su trabajo y lo evaluará. Necesita conocer los criterios a seguir en su evaluación y cuenta con un formulario para realizarla. El formulario contiene unas preguntas cerradas, que obligan al tutor a dar respuestas precisas y cuantificadas y evitan juicios emotivos o demasiado generales y un campo libre donde puede reflejar juicios y sugerencias. 


\begin{tabular}{|c|c|c|c|c|}
\hline \multirow{2}{*}{$\begin{array}{l}\text { Competencias en el plan de } \\
\text { estudios }\end{array}$} & \multirow{2}{*}{$\begin{array}{l}\text { Competencias específicas } \\
\text { capacidad para: }\end{array}$} & \multicolumn{3}{|c|}{ Competencias transversales } \\
\hline & & Instrumentales & Interpersonales & Sistémicas \\
\hline $\begin{array}{l}\text { 1. Conseguir una visión } \\
\text { global y directa del } \\
\text { funcionamiento de un centro } \\
\text { de información. }\end{array}$ & $\begin{array}{l}\text { Identificar las unidades organizativas que se } \\
\text { integran en el centro, los servicios que presta y } \\
\text { su funcionamiento. } \\
\text { Analizar las peculiaridades del centro. } \\
\text { Identificar los procedimientos asociados a los } \\
\text { servicios que presta la unidad de información }\end{array}$ & \multirow{5}{*}{$\begin{array}{l}\text { Capacidad de } \\
\text { análisis y } \\
\text { síntesis. } \\
\text { Capacidad } \\
\text { para organizar } \\
\text { y planificar. } \\
\text { Dominio del } \\
\text { lenguaje en la } \\
\text { comunicación } \\
\text { oral y escrita. }\end{array}$} & \multirow{5}{*}{$\begin{array}{l}\text { Capacidad } \\
\text { para trabajar } \\
\text { en equipo. } \\
\text { Razonamiento } \\
\text { crítico. }\end{array}$} & \multirow{5}{*}{$\begin{array}{l}\text { Motivación } \\
\text { por la } \\
\text { calidad. }\end{array}$} \\
\hline $\begin{array}{l}\text { 2. Adquirir la capacidad } \\
\text { necesaria para el desempeño } \\
\text { de una labor profesional en el } \\
\text { marco de los servicios } \\
\text { propios de bibliotecas, } \\
\text { archivos o centros de } \\
\text { documentación. }\end{array}$ & & & & \\
\hline $\begin{array}{l}\text { 3. Conocer y familiarizarse } \\
\text { con las rutinas de trabajo en } \\
\text { los procesos de recuperación, } \\
\text { localización, tratamiento y } \\
\text { difusión de información. }\end{array}$ & $\begin{array}{l}\text { Analizar las herramientas de búsqueda del } \\
\text { centro así como su funcionamiento. } \\
\text { Llevar a cabo la recuperación de información } \\
\text { (procedente de diferentes recursos: bases de } \\
\text { datos, www, blogs...) y su evaluación, con } \\
\text { arreglo a la tipología y necesidades del centro. } \\
\text { Analizar los recursos informativos } \\
\text { (documentos) según sus diferentes tipologías, } \\
\text { identificando, describiendo, sintetizando y } \\
\text { representando su contenido, con arreglo a las } \\
\text { normas, la tipología y las necesidades del } \\
\text { centro. }\end{array}$ & & & \\
\hline \multicolumn{2}{|l|}{$\begin{array}{l}\text { 4. Saber trabajar en equipo, } \\
\text { integrándose en un grupo o } \\
\text { colectivo profesional del área } \\
\text { (competencia recogida entre } \\
\text { las competencias } \\
\text { transversales). }\end{array}$} & & & \\
\hline $\begin{array}{l}\text { 5. Atender directamente a los } \\
\text { usuarios en sus necesidades } \\
\text { de información. }\end{array}$ & $\begin{array}{l}\text { Analizar e interpretar las necesidades y } \\
\text { expectativas de los usuarios y plantear una } \\
\text { estrategia de búsqueda. } \\
\text { Resolver consultas en un servicio de } \\
\text { referencia tradicional y/o virtual. } \\
\text { Elaborar productos bibliográficos. } \\
\text { Prestar apoyo a los usuarios para que definan } \\
\text { sus necesidades documentales y culminen con } \\
\text { éxito sus búsquedas. }\end{array}$ & & & \\
\hline
\end{tabular}

Tabla I. Competencias transversales y específicas desarrolladas a partir de las competencias y resultados de aprendizaje señalados en el plan de estudios. 


\begin{tabular}{|c|c|c|}
\hline Sistema de evaluación & Competencias específicas - capacidad para: & Competencias transversales \\
\hline $\begin{array}{l}\text { MEMORIA DEL ALUMNO } \\
\text { Redactor: Alumno } \\
\text { RESPONSABLE DE LA } \\
\text { EVALUACIÓN: } \\
\text { Tutor académico }\end{array}$ & $\begin{array}{l}\text { Identificar las unidades organizativas que se integran en } \\
\text { el centro, los servicios que presta y su funcionamiento. } \\
\text { Analizar las peculiaridades del centro. } \\
\text { Identificar los procedimientos asociados a los servicios } \\
\text { que presta la unidad de información. } \\
\text { Analizar las herramientas de búsqueda del centro así } \\
\text { como su funcionamiento. } \\
\text { Analizar e interpretar las necesidades y expectativas de } \\
\text { los usuarios. }\end{array}$ & $\begin{array}{l}\text { Capacidad de análisis y } \\
\text { síntesis. } \\
\text { Dominio del lenguaje en la } \\
\text { comunicación escrita. } \\
\text { Razonamiento crítico. }\end{array}$ \\
\hline $\begin{array}{l}\text { INFORME DEL TUTOR } \\
\text { Redactor: Tutor profesional } \\
\text { RESPONSABLE DE LA } \\
\text { EVALUACIÓN: } \\
\text { Tutor profesional }\end{array}$ & $\begin{array}{l}\text { Llevar a cabo la recuperación de información } \\
\text { (procedente de diferentes recursos: bases de datos, } \\
\text { www, blogs...) y su evaluación, con arreglo a la } \\
\text { tipología y necesidades del centro. } \\
\text { Analizar los recursos informativos (documentos) según } \\
\text { sus diferentes tipologías, identificando, describiendo, } \\
\text { sintetizando y representando su contenido, con arreglo a } \\
\text { las normas, la tipología y las necesidades del centro. } \\
\text { Resolver consultas en un servicio de referencia } \\
\text { tradicional y/o virtual. } \\
\text { Elaborar productos bibliográficos. } \\
\text { Prestar apoyo a los usuarios para que definan sus } \\
\text { necesidades documentales y culminen con éxito sus } \\
\text { búsquedas. }\end{array}$ & $\begin{array}{l}\text { Dominio del lenguaje en la } \\
\text { comunicación oral. } \\
\text { Trabajo en equipo. } \\
\text { Motivación por la calidad. } \\
\text { Capacidad para organizar y } \\
\text { planificar. }\end{array}$ \\
\hline
\end{tabular}

Tabla II. Competencias específicas y transversales asociadas al ámbito de verificación.

\subsubsection{La matriz de evaluación.}

Identificadas las competencias, las actividades, los actores y las responsabilidades, ha sido necesario elaborar un instrumento que permita valorar el grado de adquisición de las competencias. Para este fin se ha confeccionado una matriz que describe, para cada competencia general, sus competencias específicas y transversales con sus indicadores que, a su vez, cuentan con descripciones de las prestaciones, subdivididas en cuatro niveles, que proporcionan unos juicios sintéticos acerca de los diferentes grados de dominio.

Finalmente, para facilitar la comprensión de las tareas que compiten a cada uno de los actores y para que cada uno se centre en la tarea que le corresponde, se ha organizado la matriz de evaluación agrupando las competencias según su ámbito de puesta en práctica y verificación. A partir de estas agrupaciones se han atribuido los valores porcentuales con arreglo a las consignas del plan de estudios (ver Tablas III, IV, V, VI y VII).

\begin{tabular}{|c|c|c|c|c|}
\hline \multicolumn{5}{|c|}{$\begin{array}{l}\text { ASISTENCIA } 10 \% \\
\text { (Más de } 3 \text { faltas de asistencia sin justificar invalidará la asignatura) }\end{array}$} \\
\hline $\begin{array}{l}\text { ACTIVIDAD } \\
\text { ASOCIADA }\end{array}$ & Puntuación: 1 & Puntuación: 2 & Puntuación: 3 & Puntuación: 4 \\
\hline $\begin{array}{l}\text { Control diario de } \\
\text { firmas y puntualidad. }\end{array}$ & $\begin{array}{l}3 \text { faltas } \sin \\
\text { justificación adecuada } \\
2 \% \text {. }\end{array}$ & $\begin{array}{l}1 \text { a } 2 \text { falta } \sin \\
\text { justificación adecuada } \\
4 \% \text {. }\end{array}$ & $\begin{array}{l}\text { Solo algunas faltas } \\
\text { de puntualidad } \\
6 \% \text {. }\end{array}$ & $\begin{array}{l}\text { Sin faltas } \\
10 \%\end{array}$ \\
\hline
\end{tabular}

Tabla III. Matriz de evaluación de la asistencia del alumno. 


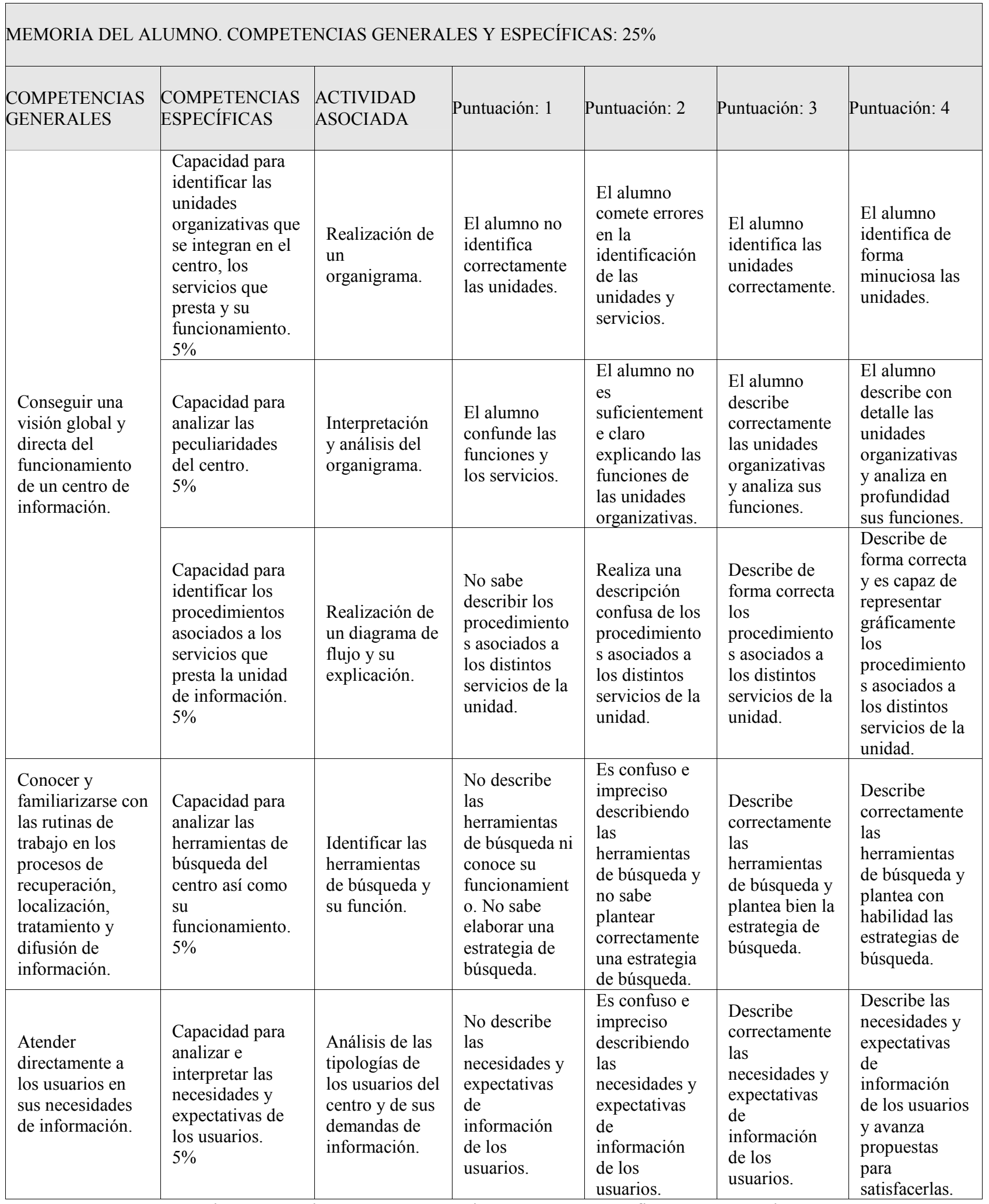

Tabla IV. Matriz de evaluación de las competencias generales y específicas de la memoria del alumno. 


\begin{tabular}{|c|c|c|c|c|c|}
\hline $\begin{array}{l}\text { COMPETENCIAS } \\
\text { [RANSVERSALES }\end{array}$ & $\begin{array}{l}\text { ACTIVIDAD } \\
\text { ASOCIADA }\end{array}$ & Puntuación: 1 & Puntuación: 2 & Puntuación: 3 & Puntuación: 4 \\
\hline $\begin{array}{l}\text { Capacidad de análisis y síntesis. } \\
5 \%\end{array}$ & $\begin{array}{l}\text { Memoria en } \\
\text { su conjunto. }\end{array}$ & $\begin{array}{l}\text { La memoria } \\
\text { resulta demasiado } \\
\text { genérica y poco } \\
\text { elaborada. }\end{array}$ & $\begin{array}{lr}\text { Algunas partes } \\
\text { de la memoria } \\
\text { necesitan una } \\
\text { mayor } & \\
\text { elaboración } & \text { por } \\
\text { parte } & \text { del } \\
\text { alumno. } & \end{array}$ & $\begin{array}{lr}\text { Se aprecia } & \text { una } \\
\text { buena labor } & \text { de } \\
\text { análisis } & \text { y } \\
\text { síntesis } & \text { por } \\
\text { parte } & \text { del } \\
\text { alumno en } & \text { la } \\
\text { mayor parte del } \\
\text { trabajo. }\end{array}$ & $\begin{array}{l}\text { El alumno } \\
\text { demuestra en } \\
\text { todo el trabajo } \\
\text { una buena labor } \\
\text { de análisis y } \\
\text { síntesis. }\end{array}$ \\
\hline $\begin{array}{l}\text { Razonamiento crítico. } \\
5 \%\end{array}$ & $\begin{array}{l}\text { Memoria en } \\
\text { su conjunto. }\end{array}$ & $\begin{array}{l}\text { La memoria no se } \\
\text { ciñe al tema, no } \\
\text { es clara, y no se } \\
\text { centra en los } \\
\text { aspectos } \\
\text { importantes. } \\
\text { El alumno no } \\
\text { aporta ningún tipo } \\
\text { de consideración. }\end{array}$ & $\begin{array}{l}\text { La memoria se } \\
\text { ciñe al tema } \\
\text { pero no siempre } \\
\text { es clara y ni se } \\
\text { centra } \\
\text { suficientemente } \\
\text { en los aspectos } \\
\text { importantes. } \\
\text { Las } \\
\text { consideraciones } \\
\text { del alumno son } \\
\text { pobres. }\end{array}$ & $\begin{array}{l}\text { La memoria se } \\
\text { ciñe al tema, es } \\
\text { clara y se centra } \\
\text { en los aspectos } \\
\text { importantes. } \\
\text { Aporta } \\
\text { consideraciones } \\
\text { personales. }\end{array}$ & $\begin{array}{l}\text { La memoria se } \\
\text { ciñe al tema, es } \\
\text { clara y se centra } \\
\text { en los aspectos } \\
\text { importantes. } \\
\text { Además } \\
\text { evidencia los } \\
\text { aspectos } \\
\text { complejos e } \\
\text { interrelacionado } \\
\text { s y sus } \\
\text { consideraciones } \\
\text { son originales. }\end{array}$ \\
\hline $\begin{array}{l}\text { Dominio del lenguaje en la } \\
\text { comunicación escrita. } \\
5 \%\end{array}$ & $\begin{array}{l}\text { Redacción de } \\
\text { la memoria en } \\
\text { su conjunto. }\end{array}$ & $\begin{array}{lr}\text { La redacción } \\
\text { presenta errores } \\
\text { ortográficos y } \\
\text { gramaticales y no } \\
\text { es coherente. No } \\
\text { maneja ra el } \\
\text { lenguaje } \\
\begin{array}{l}\text { específico de la } \\
\text { materia. }\end{array}\end{array}$ & $\begin{array}{lr}\text { La redacción } \\
\text { presenta } \\
\text { algunos errores } \\
\text { ortográficos y } \\
\text { de gramática } \\
\text { pero } \\
\text { coherente. } \\
\text { Maneja } \\
\text { dificultad es } \\
\text { lenguaje } \\
\text { específico de la } \\
\text { materia. }\end{array}$ & $\begin{array}{l}\text { La redacción es } \\
\text { correcta y } \\
\text { coherente. } \\
\text { Maneja } \\
\text { correctamente } \\
\text { el lenguaje } \\
\text { específico de la } \\
\text { materia. }\end{array}$ & $\begin{array}{l}\text { La redacción } \\
\text { demuestra una } \\
\text { expresión } \\
\text { correcta, fluida } \\
\text { y posee un } \\
\text { lenguaje rico y } \\
\text { especializado. }\end{array}$ \\
\hline
\end{tabular}

Tabla V. Matriz de evaluación de las competencias transversales de la memoria del alumno. 


\section{INFORME DEL TUTOR DEL CENTRO. COMPETENCIAS GENERALES Y ESPECÍFICAS: 30\%}

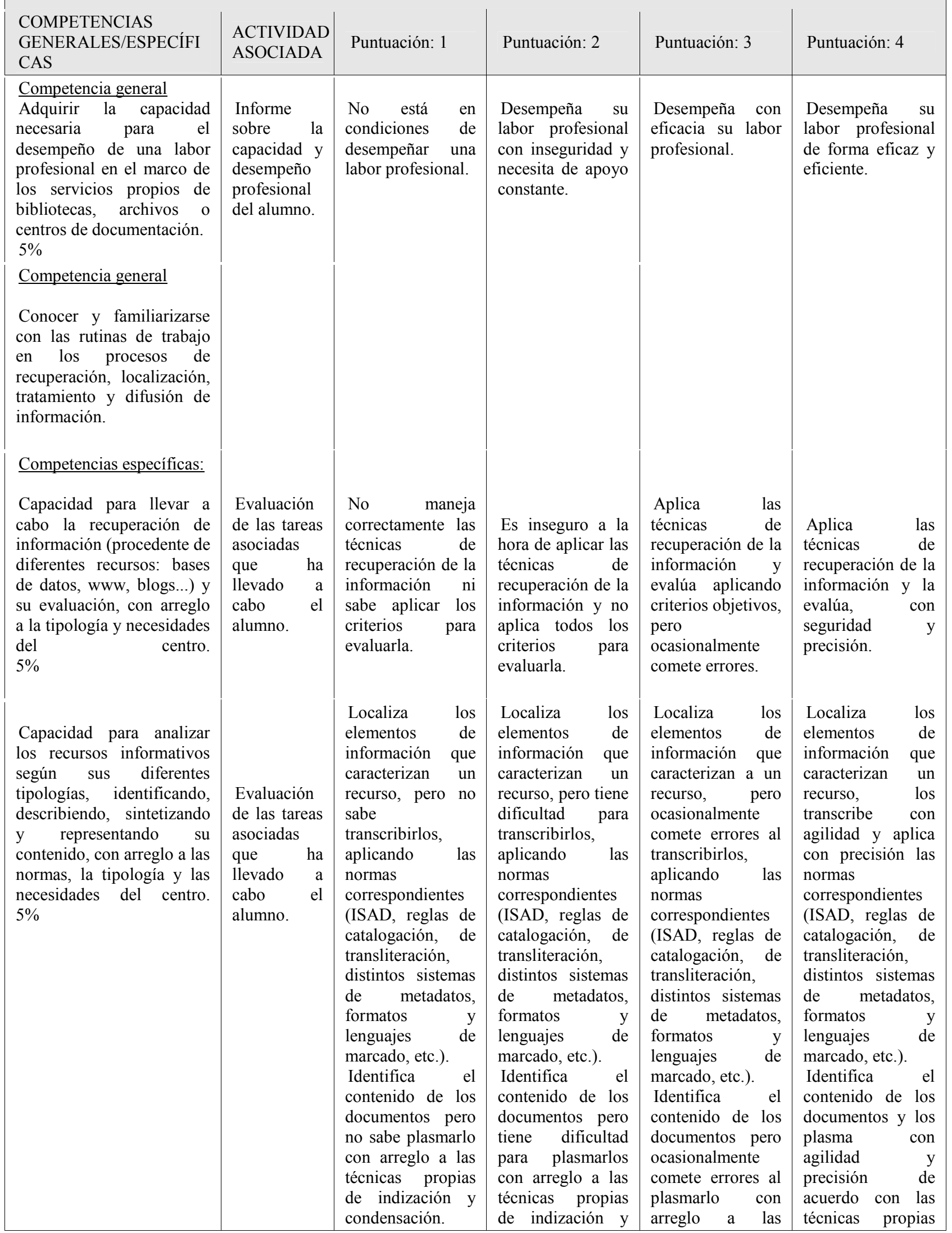




\section{INFORME DEL TUTOR DEL CENTRO. COMPETENCIAS GENERALES Y ESPECÍFICAS: 30\%}

\begin{tabular}{|c|c|c|c|c|c|}
\hline $\begin{array}{l}\text { COMPETENCIAS } \\
\text { GENERALES/ESPECÍFI } \\
\text { CAS }\end{array}$ & $\begin{array}{l}\text { ACTIVIDAD } \\
\text { ASOCIADA }\end{array}$ & Puntuación: 1 & Puntuación: 2 & Puntuación: 3 & Puntuación: 4 \\
\hline \multirow{3}{*}{\multicolumn{6}{|c|}{$\begin{array}{l}\text { Competencia general } \\
\text { Atender directamente a los } \\
\text { usuarios en } \\
\text { necesidades } \\
\text { información. }\end{array}$}} \\
\hline & & & & & \\
\hline & & & & & \\
\hline $\begin{array}{l}\text { Capacidad para resolver } \\
\text { consultas en un servicio de } \\
\text { referencia tradicional y/o } \\
\text { virtual. } \\
5 \%\end{array}$ & $\begin{array}{l}\text { Evaluación } \\
\text { de las tareas } \\
\text { asociadas } \\
\text { que } \\
\begin{array}{lr}\text { llevado } \\
\text { cabo a } \\
\text { alumno. el }\end{array}\end{array}$ & $\begin{array}{lr}\text { No conoce las } \\
\text { obras } & \text { de } \\
\text { referencia y } & \text { no } \\
\text { sabe resolver las } \\
\text { consultas } & \text { y } \\
\text { necesita siempre } \\
\text { del apoyo y ayuda } \\
\text { de } r \\
\text { compañeros. }\end{array}$ & $\begin{array}{l}\text { Conoce las obras } \\
\text { de referencia pero } \\
\text { no las maneja de } \\
\text { forma segura y a } \\
\text { menudo necesita } \\
\text { apoyo por parte } \\
\text { de los compañeros } \\
\text { para resolver las } \\
\text { consultas. }\end{array}$ & \begin{tabular}{l}
\multicolumn{2}{c}{ Conoce y maneja } \\
las obras de \\
referencia \\
resuelve \\
consultas, \\
requiriendo de \\
forma esporádica \\
la ayuda de los \\
compañeros.
\end{tabular} & $\begin{array}{l}\text { Conoce y maneja } \\
\text { con soltura las } \\
\text { obras } \\
\text { referencia y, con } \\
\text { la ayuda recibida, } \\
\text { ha aprendido a } \\
\text { resolver las } \\
\text { consultas } \\
\text { autonomía. }\end{array}$ \\
\hline $\begin{array}{l}\text { Capacidad para elaborar } \\
\text { productos de valor } \\
\text { añadido: guías de recursos, } \\
\text { bibliografias temáticas, } \\
\text { alertas bibliográficas, etc. } \\
5 \%\end{array}$ & $\begin{array}{l}\text { Evaluación } \\
\text { de las tareas } \\
\text { asociadas } \\
\text { que ha } \\
\begin{array}{lr}\text { llevado } & \text { a } \\
\text { cabo el } \\
\text { alumno. }\end{array}\end{array}$ & $\begin{array}{l}\text { No sabe elaborar } \\
\text { productos } \\
\text { bibliográficos. }\end{array}$ & $\begin{array}{l}\text { Elabora unos } \\
\text { productos } \\
\text { bibliográficos } \\
\text { poco rigurosos e } \\
\text { imprecisos. }\end{array}$ & $\begin{array}{l}\text { Desarrolla unos } \\
\text { productos } \\
\text { bibliográficos } \\
\text { correctos. }\end{array}$ & $\begin{array}{l}\text { Desarrolla } \\
\text { productos } \\
\text { bibliográficos con } \\
\text { precisión, rigor y } \\
\text { los orienta a las } \\
\text { necesidades de los } \\
\text { usuarios. }\end{array}$ \\
\hline $\begin{array}{l}\text { Capacidad para prestar } \\
\text { apoyo a los usuarios para } \\
\text { que definan sus } \\
\text { necesidades documentales } \\
\text { y culminen con éxito sus } \\
\text { búsquedas. } \\
5 \%\end{array}$ & $\begin{array}{l}\text { Evaluación } \\
\text { de las tareas } \\
\text { asociadas } \\
\text { que ha } \\
\text { llevado a } \\
\text { cabo el } \\
\text { alumno. }\end{array}$ & $\begin{array}{l}\text { No sabe ayudar a } \\
\text { los usuarios en la } \\
\text { definición de sus } \\
\text { necesidades ni } \\
\text { sabe explicar el } \\
\text { funcionamiento } \\
\text { de las } \\
\text { herramientas de } \\
\text { búsqueda. }\end{array}$ & $\begin{array}{lr}\text { Ayuda } & \text { con } \\
\text { dificultad a los } & \text { los } \\
\text { usuarios para } & \text { que } \\
\text { definan } & \text { sus } \\
\text { necesidades y } & \text { no } \\
\text { explica } & \\
\text { correctamente el } \\
\text { funcionamiento } \\
\text { de las } \\
\text { herramientas de } \\
\text { búsqueda. }\end{array}$ & $\begin{array}{l}\text { Ayuda a los } \\
\text { usuarios para que } \\
\text { definan sus } \\
\text { necesidades } \\
\text { aunque a veces } \\
\text { comete algún } \\
\text { error y explica el } \\
\text { funcionamiento } \\
\text { de las } \\
\text { herramientas de } \\
\text { búsqueda pero sin } \\
\text { que los usuarios } \\
\text { se muestren } \\
\text { siempre } \\
\text { satisfechos ante } \\
\text { los resultados } \\
\text { obtenidos. }\end{array}$ & $\begin{array}{l}\text { Ayuda a los } \\
\text { usuarios a definir } \\
\text { sus necesidades } \\
\text { documentales } \\
\text { haciendo } \\
\text { apliquen que } \\
\text { correctamente las } \\
\text { herramientas de } \\
\text { búsqueda y se } \\
\text { muestran siempre } \\
\text { satisfechos al } \\
\text { culminar con éxito } \\
\text { las mismas. }\end{array}$ \\
\hline
\end{tabular}

Tabla VI. Matriz de evaluación de las competencias generales y específicas del informe del tutor del centro. 


\begin{tabular}{|c|c|c|c|c|}
\hline \multicolumn{5}{|c|}{ INFORME DEL TUTOR DEL CENTRO. COMPETENCIAS TRANSVERSALES: 20\% } \\
\hline $\begin{array}{l}\text { COMPETENCIAS } \\
\text { TRANSVERSALES }\end{array}$ & Puntuación: 1 & Puntuación: 2 & Puntuación: 3 & Puntuación: 4 \\
\hline $\begin{array}{l}\text { Dominio del lenguaje } \\
\text { en la comunicación } \\
\text { oral. } \\
5 \%\end{array}$ & $\begin{array}{l}\text { Se expresa sin fluidez, } \\
\text { comete errores } \\
\text { gramaticales y resulta } \\
\text { confuso. No maneja el } \\
\text { lenguaje específico de } \\
\text { la materia. }\end{array}$ & $\begin{array}{l}\text { No se expresa con } \\
\text { fluidez y comete } \\
\text { errores, sin embargo } \\
\text { sus razonamientos son } \\
\text { coherentes. } \\
\text { Maneja con dificultad } \\
\text { el lenguaje específico } \\
\text { de la materia. }\end{array}$ & $\begin{array}{l}\text { Se expresa con fluidez, } \\
\text { es gramaticalmente } \\
\text { correcto y coherente. } \\
\text { Maneja correctamente } \\
\text { el lenguaje específico } \\
\text { de la materia. }\end{array}$ & $\begin{array}{l}\text { Se expresa con fluidez, } \\
\text { argumenta con soltura } \\
\text { y posee un lenguaje } \\
\text { rico y especializado. }\end{array}$ \\
\hline $\begin{array}{l}\text { Trabajo en equipo. } \\
5 \%\end{array}$ & $\begin{array}{l}\text { Se ha detectado } \\
\text { individualismo y una } \\
\text { carencia de aptitudes } \\
\text { para el trabajo en } \\
\text { equipo, así como una } \\
\text { falta importante de } \\
\text { implicación en el } \\
\text { grupo de trabajo. }\end{array}$ & $\begin{array}{l}\text { Hay poca implicación } \\
\text { en el grupo de trabajo } \\
\text { y el trabajo en equipo } \\
\text { ha sido mínimo. }\end{array}$ & $\begin{array}{l}\text { Sabe trabajar en } \\
\text { equipo y se ha } \\
\text { implicado en el grupo } \\
\text { de trabajo. }\end{array}$ & $\begin{array}{l}\text { Sabe trabajar muy bien } \\
\text { en equipo y ha } \\
\text { demostrado muy buena } \\
\text { implicación en el } \\
\text { grupo de trabajo. }\end{array}$ \\
\hline $\begin{array}{l}\text { Motivación por la } \\
\text { calidad. } \\
5 \%\end{array}$ & $\begin{array}{l}\text { Es impreciso y no } \\
\text { respeta los tiempos en } \\
\text { la realización de un } \\
\text { proyecto. }\end{array}$ & $\begin{array}{l}\text { Realiza la tarea } \\
\text { correctamente pero no } \\
\text { respeta los tiempos } \\
\text { fijados para su } \\
\text { realización. }\end{array}$ & $\begin{array}{l}\text { Realiza la tarea con } \\
\text { suficiente precisión y } \\
\text { puntualidad. }\end{array}$ & $\begin{array}{l}\text { Realiza la tarea de } \\
\text { forma sobresaliente y } \\
\text { es puntual en su } \\
\text { cumplimiento y en } \\
\text { ocasiones se adelanta a } \\
\text { los tiempos } \\
\text { establecidos. }\end{array}$ \\
\hline $\begin{array}{l}\text { Capacidad para } \\
\text { organizar y planificar } \\
5 \%\end{array}$ & $\begin{array}{l}\text { No sabe organizar } \\
\text { productos y servicios } \\
\text { en función del tipo y } \\
\text { de las necesidades del } \\
\text { centro. }\end{array}$ & $\begin{array}{l}\text { Organiza } \\
\text { dificultad con } \\
\text { productos y servicios } \\
\text { en función del tipo y } \\
\text { las necesidades del } \\
\text { centro. }\end{array}$ & $\begin{array}{l}\text { Organiza de forma } \\
\text { regular y con algún } \\
\text { error los productos y } \\
\text { servicios en función } \\
\text { del tipo y las } \\
\text { necesidades del centro. }\end{array}$ & $\begin{array}{lr}\text { Organiza } & \\
\text { correctamente } & \text { las } \\
\text { funciones, } & \text { los } \\
\text { procesos, } & \text { las } \\
\text { actividades y las } \\
\text { operaciones en función } \\
\text { del tipo y las } \\
\text { necesidades del centro. }\end{array}$ \\
\hline
\end{tabular}

Tabla VII. Matriz de evaluación de las competencias transversales del informe del tutor del centro.

\subsubsection{La evaluación de la percepción y el resultado de la experiencia.}

La evaluación es una etapa esencial para la gestión eficaz del Practicum, en cuanto que permite verificar si se han alcanzado los objetivos fijados y debe además permitir comprender las claves del éxito o del fracaso para orientar las futuras actuaciones.

La matriz, como hemos visto, es, tal como está estructurado nuestro Practicum, el instrumento principal para evaluar el aprendizaje del alumno y el que permite poner en relación objetivos y resultados desde la perspectiva de las competencias y determina el resultado objetivo que el alumno verá reflejado en su expediente.

Sin embargo esta evaluación de por sí esencial sería insuficiente si no estuviera acompañada por una evaluación de la satisfacción, no solo del alumno, sino también del centro que lo ha acogido en la figura del tutor que allí ha seguido su aprendizaje.

La satisfacción constituye una percepción individual de la experiencia que refleja el punto de vista de alumnos y tutores profesionales acerca de las prácticas, puede permitir actuaciones puntuales cuando se detecta un problema y puede representar un punto de partida para evaluaciones más complejas. En la universidad ya estamos acostumbrados a las encuestas de satisfacción que se realizan en la recta final de los cuatrimestres, sin embargo en este caso el esquema tradicional de evaluación no se adapta a la asignatura y demanda de un enfoque diferente y una realización en itinere, dirigida a monitorizar diferentes etapas del Practicum. 
Para este fin se han elaborado unos cuestionarios dirigidos a alumnos y tutores profesionales que solicitan su valoración acerca de los aspectos cualitativos y organizativos que hemos considerado más relevantes para evidenciar la calidad de las diferentes etapas y del conjunto de las prácticas. Alumnos y tutores son llamados a contestar a tres encuestas.

El objetivo fijado para la primera que se realiza una vez trascurrido un mes de estancia es que el estudiante describa en una escala ascendente de 1 a 5 su satisfacción acerca de la inducción recibida a su llegada al centro, su conformidad con las tareas que le son encomendadas y la adecuación de sus conocimientos para hacer frente a estas tareas. Por su parte el tutor debe expresar su satisfacción acerca de la actitud del alumno, valorar sus conocimientos y su capacidad para aplicarlos a las tareas que tiene que realizar.

En la segunda encuesta se solicita al alumno que valore la conformidad de las tareas que realiza con el plan de trabajo estipulado, si considera que sus conocimientos de partida se están afianzando a través de las prácticas, si se está integrando en el ambiente de trabajo, si considera correcto el seguimiento que recibe del tutor y, finalmente, en qué medida valora la utilidad de la encuesta de seguimiento que está realizando. El tutor por su parte tiene que valorar si la evolución del alumno es adecuada, en qué medida se adapta a las diferentes tareas que se le asignan, si se integra en el servicio y cómo valora el seguimiento que se está realizando.

La tercera encuesta se realiza al final de la experiencia. A los alumnos se les pide expresar su grado de satisfacción con las prácticas, en qué medida les ha ayudado para comprender el funcionamiento de una unidad de información, para adquirir las competencias necesarias para el desempeño de una labor profesional y descubrir las posibilidades que ofrecen los estudios realizados. A los tutores se les pregunta acerca de las horas dedicadas a tutorizar al alumno, si considera adecuada la organización y el seguimiento del Practicum, el grado de satisfacción con la experiencia y, por último, si considera que el alumno reviste los conocimientos y las competencias que le hacen apto para ser contratado.

\section{RESULTADOS Y CONCLUSIONES.}

Los datos obtenidos en el conjunto de las encuestas realizadas en estos dos años evidencian unos resultados muy positivos que sintetizamos a continuación.

En la primera encuesta que se realiza al mes de iniciarse las prácticas los estudiantes expresan (con una escala ascendente de 1 a 5) que con sus conocimientos académicos la dificultad percibida para realizar las tareas alcanza de media un 1,4. Estiman la atención prestada por parte del tutor del centro con un valor de 4,5 y perciben las prácticas adecuadas al plan de formación establecido. Por su parte, los tutores valoran la actitud de los alumnos en un 4,8, sus conocimientos de partida en un 4,3 y su capacidad de aplicar los conocimientos a las características del centro en un 4,8 .

En la segunda encuesta de seguimiento los alumnos valoran con un 4,2 la capacidad de adquirir una visión general del centro que le proporcionan las tareas encomendadas. El afianzamiento de sus conocimientos de partida a través de su aplicación práctica se sitúa en un 4,1. La integración con los compañeros en un 4,7. La formación y el apoyo que reciben en el centro en un 4,5. Además consideran adecuado el seguimiento llevado a cabo por la Comisión del Practicum. Los tutores también expresan su conformidad con el seguimiento que se realiza y, por otra parte, alcanza un 4,7 tanto la evolución del alumno como su adaptación a las diferentes tareas y su integración en el servicio.

En la tercera y última encuesta que se realiza al finalizar las prácticas los estudiantes puntúan con un 4,7 su satisfacción con las mismas. Valoran con un 4,5 la utilidad del Practicum para adquirir una visión general de una unidad de información, para consolidar sus competencias y para descubrir las posibilidades que ofrecen los estudios realizados. Alcanza un 4,6 el apoyo del tutor profesional y la inducción recibida. El seguimiento y apoyo del tutor académico es valorado con un 4,4. Sin embargo la oferta de centros merece una valoración del 3,9. Los comentarios no son frecuentes y suelen ser simples expresiones de satisfacción. Los tutores valoran su satisfacción con la experiencia en un 5. La organización del Practicum les merece un 4,8 al igual que el seguimiento que se realiza. A la pregunta de si contratarían a los estudiantes la respuesta siempre es positiva. En cuanto a los comentarios que se adjuntan a las encuestas no resultan especialmente significativos ya que reiteran las expresiones de satisfacción, solo en una ocasión se sugiere la oportunidad de ampliar la duración a seis meses.

La evaluación de las memorias de los alumnos, a cargo del tutor académico, y que se realiza con una escala de 1 a 4, siendo el 4 la nota mejor, ha verificado una buena consecución de las competencias. Las notas oscilan de media entre el 3 y el 4, siendo las competencias específicas las que registran las notas más bajas. Son las relacionadas con la 
capacidad de analizar las peculiaridades del centro y familiarizarse con las rutinas de trabajo en los procesos de recuperación, localización, tratamiento y difusión de la información. Entre las competencias transversales la nota más baja la registra la capacidad de análisis y síntesis.

Los informes de los tutores profesionales asignan notas muy altas (utilizando la misma escala de 1 a 4 ) atribuyendo de media valores entre el 3,7 y el 4 a la adquisición de las competencias por los alumnos. Sin embargo, la excesiva homogeneidad de las notas y cierta discordancia con los resultados de la evaluación de la memoria realizada por los alumnos, nos hace sospechar ( en lugar de utilizar la matriz de evaluación de forma objetiva prefieren atribuir a los alumnos una evaluación de conjunto, premiando su trabajo y reflejar para cada competencia una evaluación homogeneizada que evita señalar los aspectos más débiles.

A la vista de estos resultados consideramos que el modelo de Practicum que hemos diseñado es bueno, la percepción de alumnos y tutores es positiva, pero que mejorará si para los próximos cursos, además de incrementar nuestros esfuerzos en la captación de centros para aumentar la oferta, reforzamos desde la plataforma docente el seguimiento que realiza el tutor académico. Esto se realizará promoviendo e intensificando el intercambio de impresiones a través del foro y proporcionando una mayor y mejor selección de la documentación de apoyo. Asimismo intensificaremos la relación y el intercambio de opiniones con los tutores profesionales integrándolos en la plataforma docente. Pensamos que la posibilidad de conocer nuestras actuaciones e intercambiar de forma fluida informaciones y opiniones con el tutor académico, pero también con los demás tutores profesionales y los estudiantes, hará posible enriquecer la experiencia, dinamizar las relaciones y comprender mejor la utilidad que reviste el empleo de la matriz a la hora de plasmar las evaluaciones.

NOTA.

$<$ http://www.deseco.admin.ch/bfs/deseco/en/index/02.parsys.34116.downloadList.87902.DownloadFile.tmp/oecddesecostrategypaperdeelsaedcericd 20029.pdf>.

\section{BIBLIOGRAFÍA.}

ANECA. AGENCIA NACIONAL DE EVALUACIÓN DE LA CALIDAD Y ACREDITACIÓN (España). Libro Blanco del Título de Grado en Información y Documentación. Madrid, 2004, [en línea]. Disponible en: $<$ http://www.aneca.es/var/media/150424/libroblanco_jun05_documentacion.pdf $>$. [Consulta: 24 de julio de 2013].

ARAKAKI, M. y DE LA VEGA, A. Prácticas profesionales en los planes de estudio de bibliotecología y ciencias de la información: un panorama latinoamericano. En: IFLA-Internships and placements for the new information society - Education and Training, 2011. Disponible en: $<$ http://conference.ifla.org/past/ifla77/110-arakaki-es.pdf $>$. [Consulta: 24 de julio de 2013].

BAIRD, B.N. Internship, Practicum, and Field Placement Handbook: A Guide for the Helping Professions. 5th Ed. New York: Prentice Hall, 2008.

BEARD, D.F. Assessment of Internship Experiences and Accounting Core Competencies. Accounting Education: An International Journal, 2007, vol. 16, $\mathrm{n}^{\mathrm{o}}$ 2, p. 207-220.

BOYATZIS, R. Competent Manager: a model for effective performance. New York: Wiley and Sons, 1982.

CARABAÑA MORALES, J. Competencias y Universidad, o un desajuste por mutua ignorancia. BORDÓN, Revista de Pedagogía, 2011, vol. 63, n 1, p. 15-31.

CARRIZO SAINERO, G. Las prácticas en la formación de los profesionales de Biblioteconomía y Documentación. En: Organización del Conocimiento en Sistemas de Información y Documentación, Actas del II Encuentro de ISKO-España. Getafe, 16 y 17 de noviembre. Zaragoza: Universidad, 1997, p. 169-174.

CASTRO MORERA, M. ¿Qué sabemos de la medida de las competencias? Características y problemas psicométricos en la evaluación de las competencias. BORDÓN, Revista de Pedagogía, 2011, vol. 63, n 1, p. 109-123.

CHAÍN NAVARRO, C.; MUÑOZ CAÑAVATE, A. y BLANCO FAURA, I. El Practicum en las diplomaturas de Biblioteconomía y Documentación españolas. Revista General de Información y Documentación, 2009, vol. 19, p. 145-160.

ESPELT, C. y MAÑA, T. El valor añadido de las prácticas externas para los estudiantes y las instituciones: la experiencia de la Facultad de Biblioteconomía y Documentación de Barcelona. [En línea]. En: IFLA-Internships and placements for the new information society - Education and Training, 2011. Disponible en: $<$ http://conference.ifla.org/past/ifla77/120-espelt-es.pdf $>$. [Consulta: 24 de julio de 2013]. 
FERNÁNDEZ BAJÓN, M.T. El Practicum de la Escuela Universitaria de Biblioteconomía y Documentación de la Universidad Complutense de Madrid: reflexiones de una experiencia. Documentación de las Ciencias de la Información, 1998, vol. 21, p. 131-142.

FERNÁNDEZ-SALINERO MIGUEL, C. Las competencias en el marco de la convergencia europea: Un nuevo concepto para el diseño de programas educativos. Encounters on Education, 2006, vol. 7, p. 131-153.

GONZÁLEZ, J. y WAGENAAR, R. (ed.). Tuning educational structures in Europe: Universities' contribution to the Bologna process: an introduction. Bilbao: Universidad de Deusto, 2006.

HAY/McBER. Generic Competency Dictionary. Boston: McBer\&Company, 1996.

JARA DE SÚMAR, J. y TALAVERA IBARRA, A.M. Value of the Practicum in library and information science education in Peru, [en línea]. En: IFLA-Internships and placements for the new information society - Education and Training, 2011. Disponible en: <http://conference.ifla.org/past/ifla77/120-sumar-en.pdf $>$. [Consulta: 24 de julio de 2013].

MANO GONZÁLEZ, M. y MORO CABERO, M. La evaluación por competencias: propuesta de un sistema de medida para el grado en Información y Documentación, [en línea]. BiD: Textos Universitaris de Biblioteconomia $i$ Documentació, 2009, $\mathrm{n}^{\mathrm{o}}$ 23. Disponible en: <http://bid.ub.edu/23/delamano2.htm>. [Consulta: 24 de julio de 2013].

McCLELLAND, D.C. Testing for Competencies rather than intelligence. American Psychologist, 1973, vol. 28, p. 114.

MENDO, C. et al. Valoración del Practicum por los alumnos de la Escuela Universitaria de Biblioteconomía y Documentación de la UCM. Resultados de dos encuestas. En: Homenaje a Juan Antonio Sagredo Fernández. Estudios de Bibliografía y Fuentes de Información. Madrid: Universidad Complutense de Madrid, 2001, p. 379408.

MONEDA, M. y PEIS, E. Planteamiento y desarrollo de la asignatura "Prácticas y Memoria" en la Escuela Universitaria de Biblioteconomía y Documentación de la Universidad de Granada. En: A. MARTÍN OÑATE, (ed.). VIII Jornadas Bibliotecarias de Andalucía. Huelva: Asociación Andaluza de Bibliotecarios, Diputación Provincial, 1995, p. 193-204.

OLAZ CAPITÁN, A.J.; ORTIZ GARCÍA, P. y SÁNCHEZ-MORA MOLINA, M.I. Una aproximación conceptual al término competencia desde un análisis polisémico, [en línea]. Congreso Internacional de Innovación docente. Universidad Politécnica de Cartagena, 6, 7 y 8 de julio de 2011. Disponible en: $<$ http://repositorio.bib.upct.es/dspace/bitstream/10317/2079/1/c13.pdf $>$. [Consulta: 24 de julio de 2013].

OSUNA, R. La puesta al día del Practicum en los nuevos planes de estudio del Título de Grado en Información y Documentación en las facultades españolas ante las demandas del EEES. En: FRIAS, J.A. y TRAVIESO, C. (eds.). Formación, investigación y mercado laboral en Información y Documentación en España y Portugal. Salamanca: Universidad de Salamanca, 2008, p. 203-315.

PACIOS, A.R. Assesment of the Practicum by Students from the Perspective of the Induction Process. Journal of Education for Library and Information Science, 2013, vol. 54, n 3, p. 191-204.

PIMIENTA PRIETO, J.H. Secuencias didácticas: aprendizaje y evaluación de competencias en Educación Superior. BORDÓN, Revista de Pedagogía, 2011, vol. 63, nº 1, p. 77-92.

REVISTA DE EDUCACIÓN [en línea], enero-abril 2011, $\mathrm{n}^{\circ}$ 354. La formación práctica de estudiantes universitarios: repensando el Practicum. $<$ http://www.revistaeducacion.educacion.es/re354/re354.pdf $>$. [Consulta: 24 de julio de 2013].

RIBES IÑESTA, E. El concepto de competencia: su pertinencia en el desarrollo psicológico y la educación. BORDÓN, Revista de Pedagogía, 2011, vol. 63, n 1, p. 33-45.

RYCHEN, D.S. y SALGANIK, L. Definition and Selection of Competencies (DESECO): Theoretical and Conceptual Foundations. Strategy Paper, 2002, [en línea]. Disponible en: $<$ http:/www.deseco.admin.ch/bfs/deseco/en/index/02.parsys.34116.downloadList.87902.DownloadFile.tmp/oecdd esecostrategypaperdeelsaedcericd20029.pdf $>$. [Consulta: 25 de julio de 2013].

SALVADOR OLIVÁN, J.A. et al. La presencia de competencias en información en los títulos de grado de la Universidad de Zaragoza [en línea]. Anales de Documentación, 2011, vol. 14, $\mathrm{n}^{\circ}$ 2. Disponible en: $<$ http://revistas.um.es/analesdoc/article/view/114981>. [Consulta: 24 de julio de 2013].

SARGENT, A.R.; BECKER, B.W. y KLINGBERG, S. Incorporating library school interns on academic library subject teams. The Journal of Academic Librarianship, 2011, vol. 37, nº 1, p. 28-33.

SPENCER, L.M. y SPENCER, S.M. Competence at Work. New York: John Wiley and Sons, 1993.

TEJADA ARTIGAS, C.M. et al. El diseño del plan docente en Información y Documentación acorde con el Espacio Europeo de Educación Superior: un enfoque por competencias [en línea]. Madrid: Facultad de Ciencias de la Documentación, Universidad Complutense de Madrid, 2006. Disponible en: $<$ http://eprints.ucm.es/6005/1/MANUAL.pdf $>$. [Consulta: 24 de julio de 2013]. 
TEJADA FERNÁNDEZ, J. El trabajo por competencias en el Practicum: cómo organizarlo y cómo evaluarlo [en línea]. Revista electrónica de investigación educativa, 2005, vol. 7, $\mathrm{n}^{\mathrm{o}} 2$. Disponible en: $<$ http://www.redalyc.org/articulo.oa?id=15507211>. [Consulta: 24 de julio de 2013].

UNIVERSIDAD CARLOS III DE MADRID. Programa Asignatura Prácticas en Unidades de Información, Diplomado en Biblioteconomía y Documentación, Curso 2o. Disponible en: $<$ http://www3.uc3m.es/reina/Fichas/Idioma_1/51.10428.html $>$. [Consulta: 25 de julio 2013].

UNIVERSIDAD CARLOS III DE MADRID. Programa Asignatura Prácticas en Unidades de Información, Diplomado en Biblioteconomía y Documentación, Curso $3^{\circ}$. Disponible en: $<$ http://www3.uc3m.es/reina/Fichas/Idioma_1/51.10413.html $>$. [Consulta: 25 de julio de 2013].

UNIVERSIDAD CARLOS III DE MADRID. Programa Asignatura Prácticas en Unidades de Información, Licenciado en Documentación, Curso $22^{\circ}$ Disponible en: $<$ http://www3.uc3m.es/reina/Fichas/Idioma_1/11.10612.html $>$. [Consulta: 25 de julio de 2013].

WESTBROCK, T. y FABIAN, S. Proficiencies for Instruction Librarians: Is there still a disconnect between professional education and professional responsibilities? College \& Research Libraries, 2010, vol. 71, $\mathrm{n}^{\circ}$ 6, p. 569590.

XI SYMPOSIUM INTERNACIONAL sobre el Practicum y las prácticas en empresas en la Formación Universitaria, [en línea]. Pontevedra, 27-29 de junio de 2011. Disponible en: $<$ http://webs.uvigo.es/poio/html/presentacion.htm>. [Consulta: 24 de julio de 2013].

ZABALZA BERAZA, M.A. El Practicum en la formación universitaria: estado de la cuestión. Revista de Educación, 2011, no 354, p. 21-43. 